\title{
MULTIDIMENSIONAL INTEGRAL CALCULUS VISUALIZATION
}

\author{
Corresponding Author \\ Dr. Mario Arturo RUIZ Estrada, \\ Social Security Research Centre (SSRC) \\ Centre of Poverty and Development Studies (CPDS) \\ University of Malaya, \\ Kuala Lumpur 50603, \\ [Tel] (60) 37967-3728 \\ [H/P] (60) 126850293 \\ [E-mail] marioruiz@um.edu.my
}

\begin{abstract}
The idea to write this research paper is to introduce a new multidimensional graphical approach to visualize a large number of integrals behavior in different periods of time and spaces under the application of the Megasfinity Coordinate Space. The Megasfinity Coordinate Space is willing to mapping a large number of integrals in different dimensions and periods of time extensively in the same graphical space as a whole.
\end{abstract}

Keywords: Calculus, integrals, multidimensional coordinate spaces.

JEL: C1, C2, C3

\section{Introduction}

Since the beginning of integral calculus origins from Cavalieri in the year 1635, the main contribution of Cavalieri is based on its notion about "method of indivisibles" (Harris and Stocker, 1998), this method makes reference that exist in any area a large number of parallel planar graph areas (Kern and Bland, 1948). According to Abramowitz and Stegun (1972), the formal integral calculus appears officially with Sir John Wallis from the year 1656, It is possible to observe in its master piece of research the famous Wallis Law for integration of polynomials. Moreover, we have Fermat's contribution in the integral calculus under the introduction of the integrals in infinity series and the interior extremum theorem (Hardy and Wright,1979). In fact, this research paper is proposing an alternative approach of integral calculus is entitled "The Multidimensional Integral Calculus". The Multidimensional Integral Calculus is using an alternative mathematical and graphical approach under the uses of the Megasfinity Coordinate Space. The Megasfinity Coordinate Space is based on a large number of integrals in different Spaces level (S), Mega-Spaces level (MS), General Spaces level (GS), Sub-Spaces level (SS), Micro-Spaces level (MS), and Nano-Spaces level (NS) simultaneously. Finally, the difference between the traditional integral calculus and the multidimensional integral calculus is that we are using more than 2-Dimensions and 3-Dimensions in our mathematical and graphical method of integrals. 


\section{An Introduction to the Megasfinity Coordinate Space}

The Megasfinity Coordinate Space is based on five premises: First premise, it is based on the premise that the Megasverses is multi-dimensional space, and that each part of the Megasverses is moving at different speeds of time. Additionally, in this research paper, we assume that the expression or number "Megasfinity" is equal to the infinite exponential of infinity (Ruiz Estrada, 2012)

Second premise, the Megasfinity Coordinate Space (See Figure 1) is divided by a large number of Spaces (S) in our case we are referring to Dimensions levels (D) or Mega-Spaces levels (MS). Hence, the Megasverses keeps Megasfinity number of Mega-Spaces levels (MS) (see Expression 1.1), for each Mega-Space level (MS) is formed by Megasfinity number of GeneralSpaces levels (GE) (see Expression 1.2). At the same time, in each General-Space level (GE) is built by Megasfinity number of Sub-Spaces levels (SS) (see Expression 1.3). In the next level, in each Sub-Space level (SS) is configured by Megasfinity number of Micro-Spaces levels (MS) (see Expression 1.4). Subsequently, in each Micro-Space level (MS) is built by Megasfinity number of Ji $\alpha / \beta$-Spaces areas (see Expression 1.5), and finally in each Nano-Space level (NS) is based on Megasfinity number of coordinate interceptions between JI $\alpha$-Spaces levels and JI $\beta$-Spaces levels (see Expression 1.6). In fact, all S, MS, GS, SS, MS, and NS are moving in real time (济) and apply the multi-integrated principle (㓠) together.

This is to join all Spaces levels (S) in the Megasfinity Coordinate Space simultaneously. Finally, the Megasfinity coordinate space is based on the integration of all Spaces levels (S), Mega-Spaces levels (MS), General-Spaces levels (GS), Sub-Spaces levels (SS), Micro-Spaces level (MS), and Nano-Spaces levels (NS).

$$
\begin{aligned}
& \mathrm{S}=\left\{\mathrm{MS}_{0}, \mathrm{MS}_{1}, \ldots, \mathrm{M}_{\infty}\right\} \\
& \mathrm{MS}=\left\{\mathrm{GS}_{0}, \mathrm{GS}_{1}, \ldots, \mathrm{G}_{\infty}\right\} \\
& \mathrm{GS}=\left\{\mathrm{SS}_{0}, \mathrm{SS}_{1}, \ldots, \mathrm{SS}_{\infty}\right\} \\
& \mathrm{SS}=\left\{\mathrm{MS}_{0}, \mathrm{MS}_{1}, \ldots, \mathrm{MS}_{\infty}\right\} \\
& \mathrm{MS}=\left[\left(\mathrm{JI}_{\alpha}-\mathrm{Spaces}_{0} \times \mathrm{JI}_{\beta}-\text { Spaces }_{0}\right) \ldots\left(\mathrm{JI}_{\alpha}-\text { Spaces }_{\infty} \times \mathrm{JI}_{\beta}-\text { Spaces }_{\infty}\right)\right] \ldots
\end{aligned}
$$

NS-Space $=\left[\left(\mathrm{JI}_{\alpha}-\right.\right.$ Spaces $_{0}, \mathrm{JI}_{\beta}-$ Spaces $\left._{0}\right) \ldots\left(\mathrm{JI}_{\alpha}-\right.$ Spaces $\left.\left._{\infty}, \mathrm{JI}_{\beta}-\mathrm{Spaces}_{\infty}\right)\right]$

Therefore, the Megasfinity Coordinate Space starts from dimension level " 0 " (see Expression 1.7) to dimension level " $\infty$ " (see Expression 1.8):

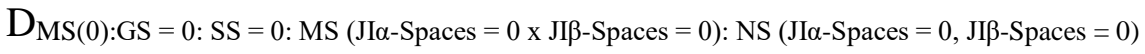

$\mathrm{D}_{\mathrm{MS}}(\infty): \mathrm{GS}=\infty: \mathrm{SS}=\infty: \mathrm{MS}=\infty: \mathrm{NS}(\mathrm{JI} \alpha-$ Spaces $=\infty \mathrm{x} \mathrm{JI} \beta-$ Spaces $=\infty): \mathrm{NS}(\mathrm{JI} \alpha-$-Spaces $=\infty \mathrm{x} \mathrm{JI} \beta-$ Spaces $=\infty)$

The final general coordinate structure to analysing the Megasfinity Coordinate Space is demonstrated by Expression 1.9:

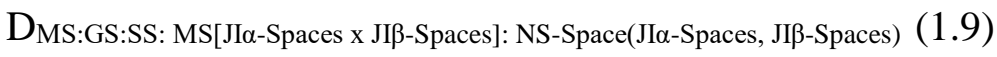

The third premise is that the Nano-Space level (NS) is a Nano-coordinate system $\left(\mathrm{JI}_{\alpha}, \mathrm{JI}_{\beta}\right)$ in its Sub-Space level (SS). In the case of each Micro-Space level (MS) is a specific area between JI $\alpha$-sub-coordinate level (base) and $\mathrm{JI}_{\beta}$-sub-coordinate level (height) within its Sub-Space level (SS) respectively (see Expression 1.5). 
The fourth premise is that the Nano-Space level (NS) shows the interception between $\mathrm{JI}_{\alpha^{-}}$ Spaces $\wedge \mathrm{JI}_{\beta}$-Spaces. Hence, the Nano-Space level (NS) is a rigid body (or point) that is simply hanging or unfolding in its respective Sub-Space level (SS); in addition, the Nano-Space level (NS) cannot occupy the same space at the same time. If we apply vectors among $\mathrm{JI}_{\alpha}$-Spaces $\wedge$ $\mathrm{JI}_{\beta}$-Spaces together in the same Micro-Space level (MS), then we can visualize a non-linear curve moving in real time (-) in its Sub-Space level (SS).

The fifth premise is that the Megasfinity Coordinate Space (see Figure 1) is running under different speeds and type of times. Initially, we have four types of time ( $\mathrm{t}$ ): constant time (past time $=\partial \mathrm{t}-1)$, partial time (present time $=\partial$ to), chaos time (future time $\partial-V_{t}$ ) and general time (GT) (see Expression 1.10). General time is the synchronization at the same time of all MegaSpaces levels, General-Spaces levels, Sub-Spaces levels, Micro-Spaces levels, and NanoSpaces levels within the same Megasfinity Coordinate Space. Additionally, all systems in the Megasverses are running at different speeds of time; these depend on the location of each dimension level or space level and the distance from the point of origin.

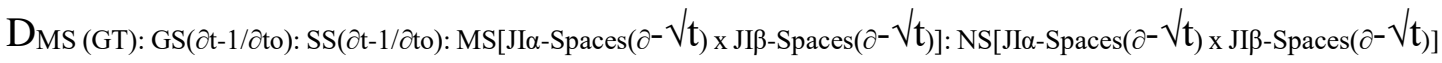

\section{An Introduction to the Multidimensional Integral Calculus:}

Always, the idea about integration is define as the anti-differentiation (anti-derivative) in certain specific period of time and space according to the integral calculus. The uses of the classic $f(x)$ that offer appropriate information of a constant that shows different behaviour in the process of integration. Hence, the primitive function $\mathrm{f}(\mathrm{X})$ keeps different and infinite number of possible combinations by using integration. In our case, the expression

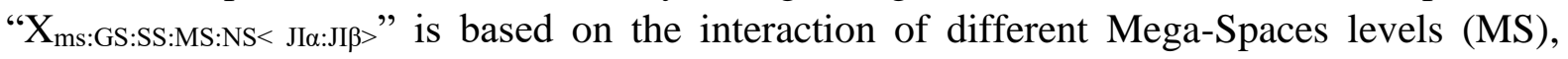
General-Spaces levels (GS), Sub-Spaces levels (SS), Micro-spaces levels (MS), Nano-Spaces levels (NS), and $\mathrm{JI}_{\alpha}$-Spaces levels $\wedge \mathrm{JI}_{\beta}$-Spaces levels in different Spaces levels (S) or Dimensions levels simultaneously. Therefore, we have a new configuration of

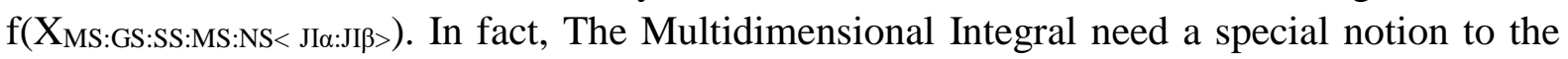

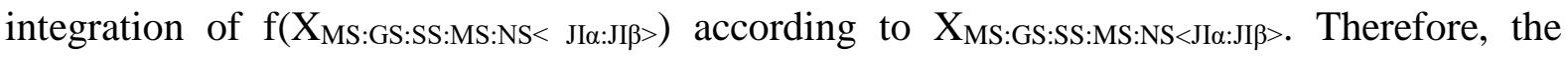
integral sign we are including from top the Mega-Spaces levels (MS), General-Spaces levels (GS), Sub-Spaces levels (SS), Micro-spaces levels (MS), until to arrive to the bottom with its Nano-Space level (NS). Now, we have opportunity to identified any integral in different Dimension(s) level(s) based on the location of the Mega-Space level (MS), General-Space level (GS), Sub-Space level (SS), Micro-space level (MS), Nano-Space level (NS) respectively. The

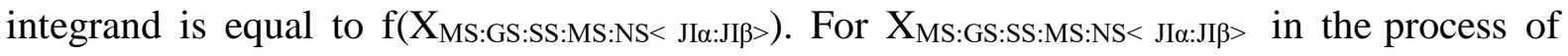

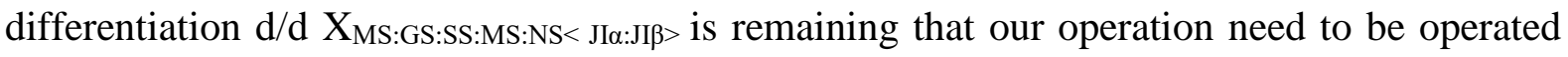

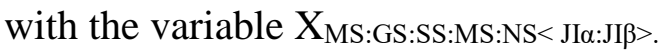

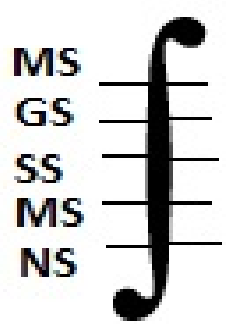

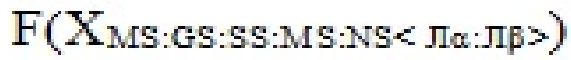




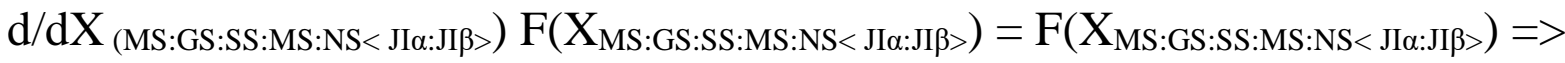

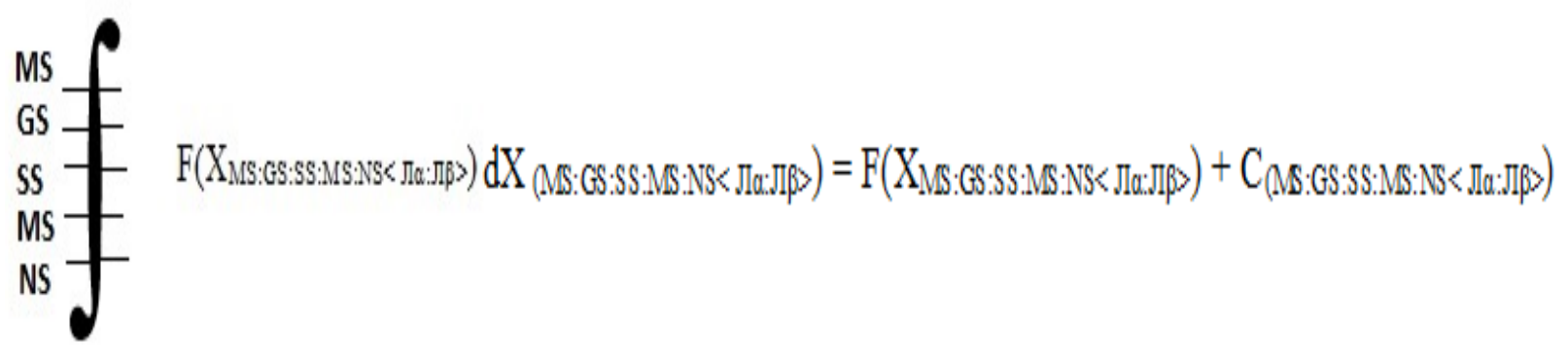

According to expression 2, we available to reverse the multidimensional differentiation process that give result to the multidimensional differential. The basic rules of multidimensional integration are very similar to the classic rules of integration. In the case of rules in the multidimensional integration is using the variable X with its Mega-Space level (MS), GeneralSpace level (GS), Sub-Space level (SS), Micro-space level (MS), Nano-Space level (NS), and $\mathrm{JI}_{\alpha}$-Space level $\wedge \mathrm{JI}_{\beta}$-Space level respectively. The rules in the multidimensional integration is nine rules such as (i) the multidimensional power rule (Expression 3); (ii) the multidimensional exponential rule (Expression 4); (iii) the multidimensional logarithmic rule (Expression 5); (iv) the multidimensional integral of sum (Expression 6); (v) the multidimensional integral of a multiple (Expression 7); (vi) multidimensional substitution rule (Expression 8); (vii) multidimensional integration by parts (Expression 9). In our case, we have nine rules of integration in the rules of multidimensional integration. We have two extra rules are (viii) the dimension viability inter-connection (Expression 10) and (ix) normalization interconnectivity rule (Expression 11).

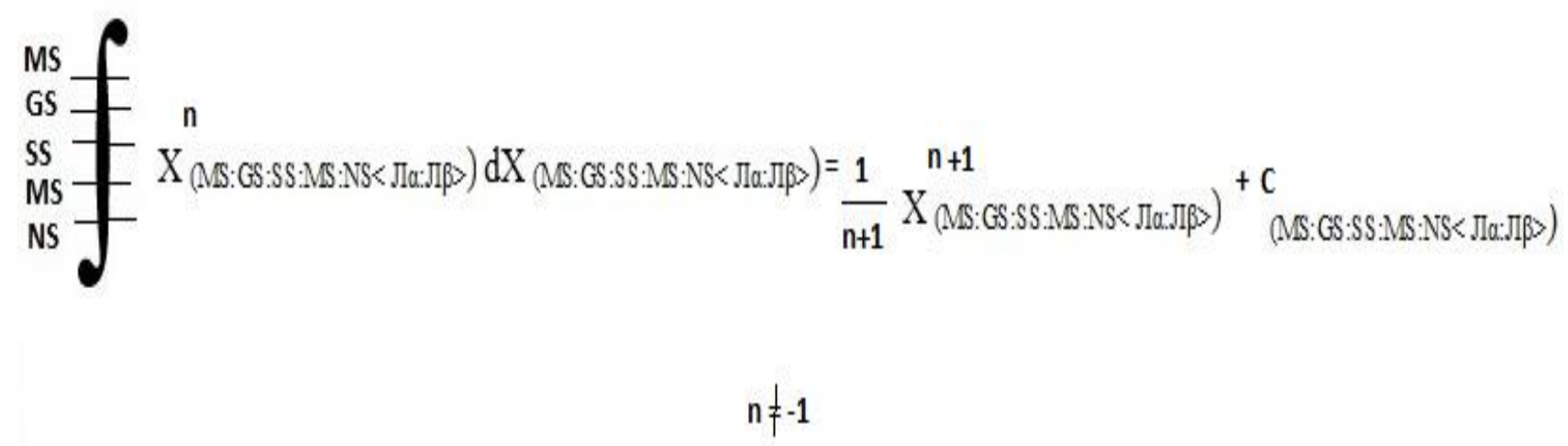



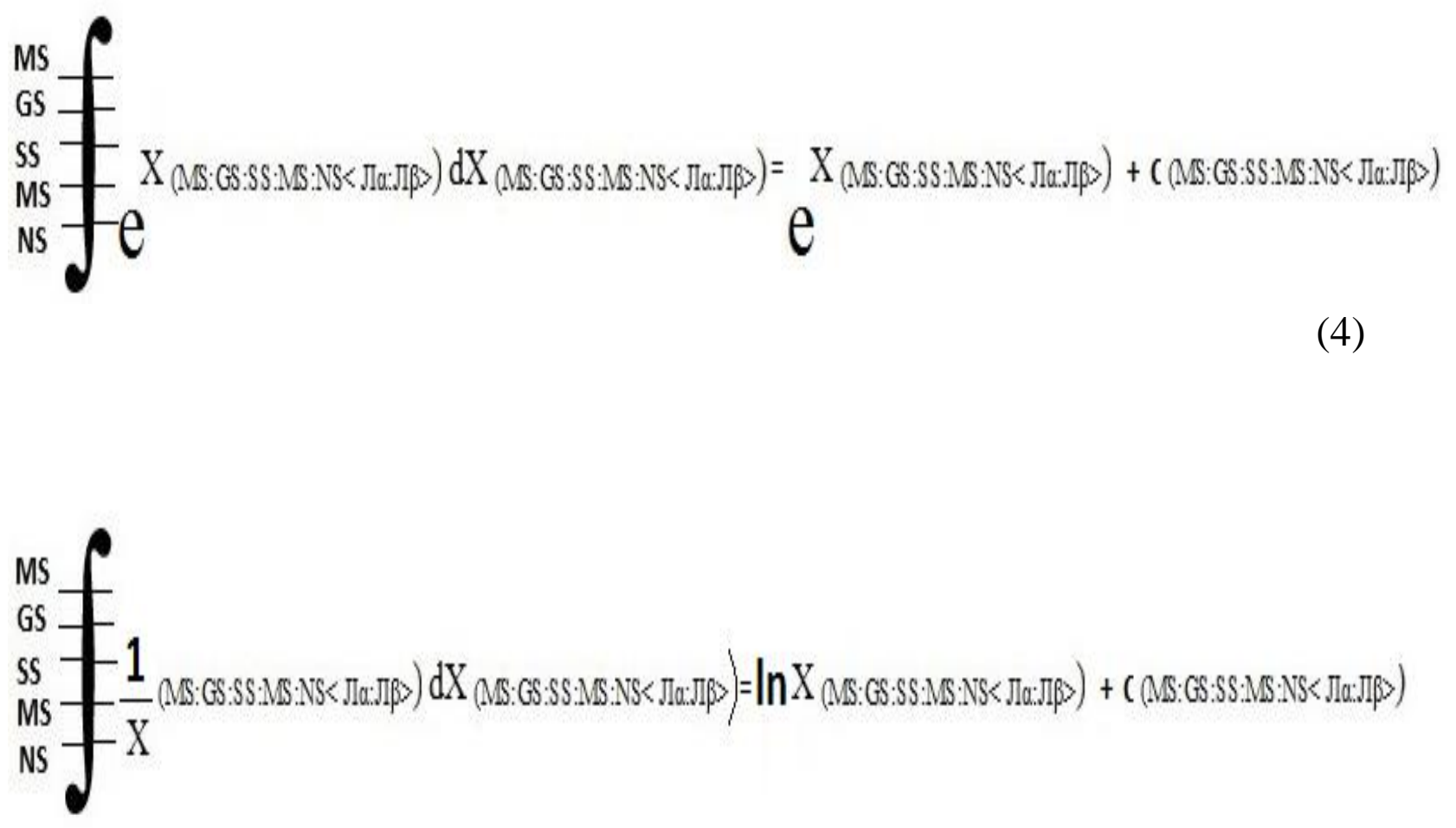

(5)
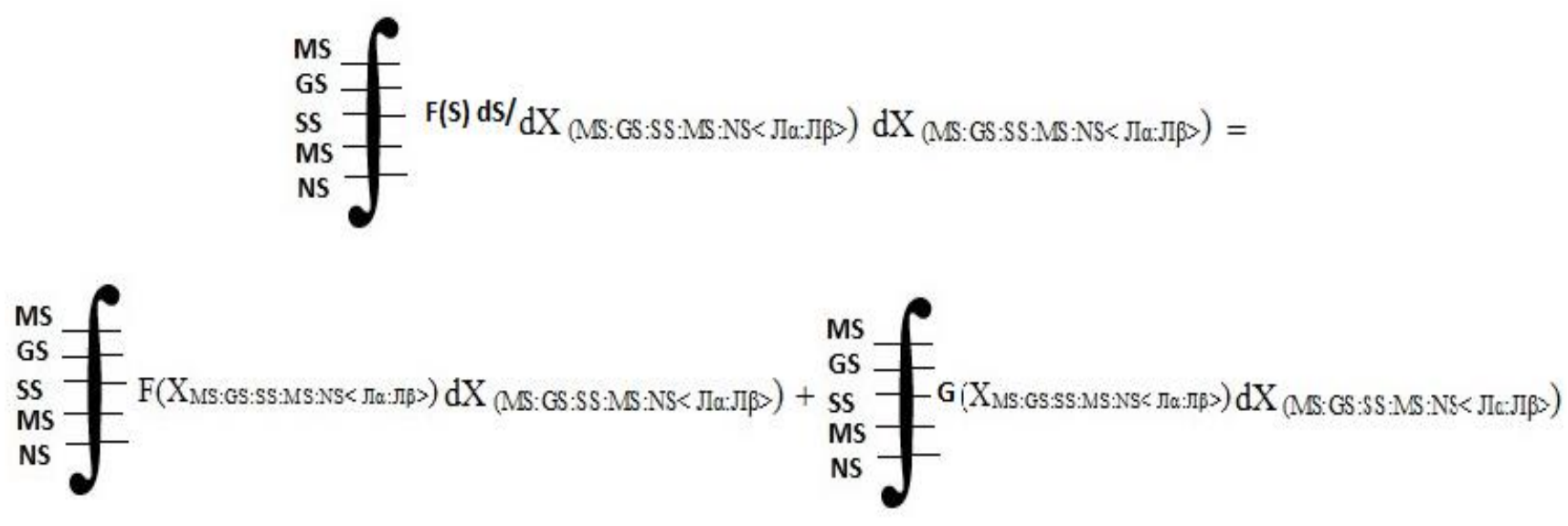

(6) 

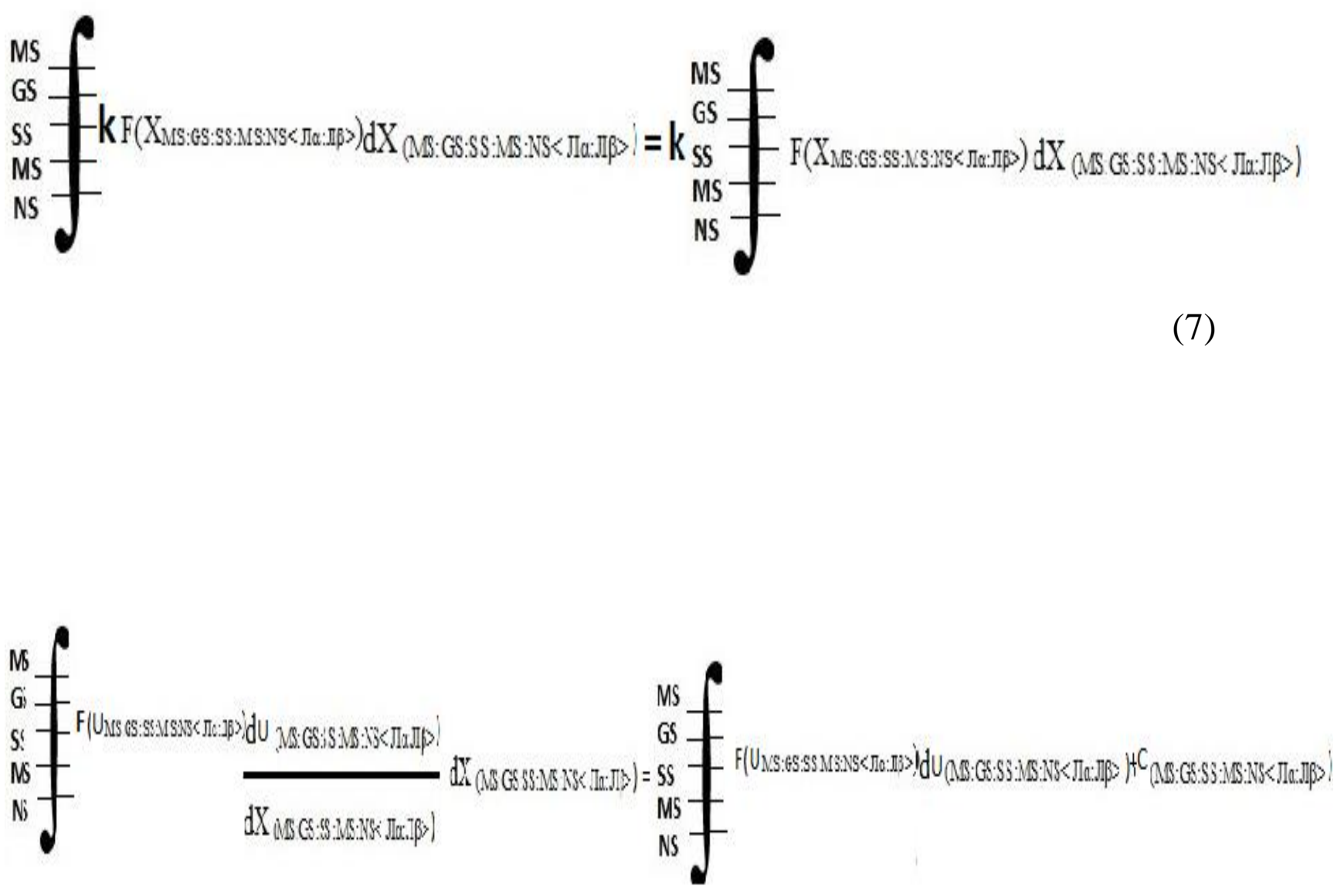

(8)

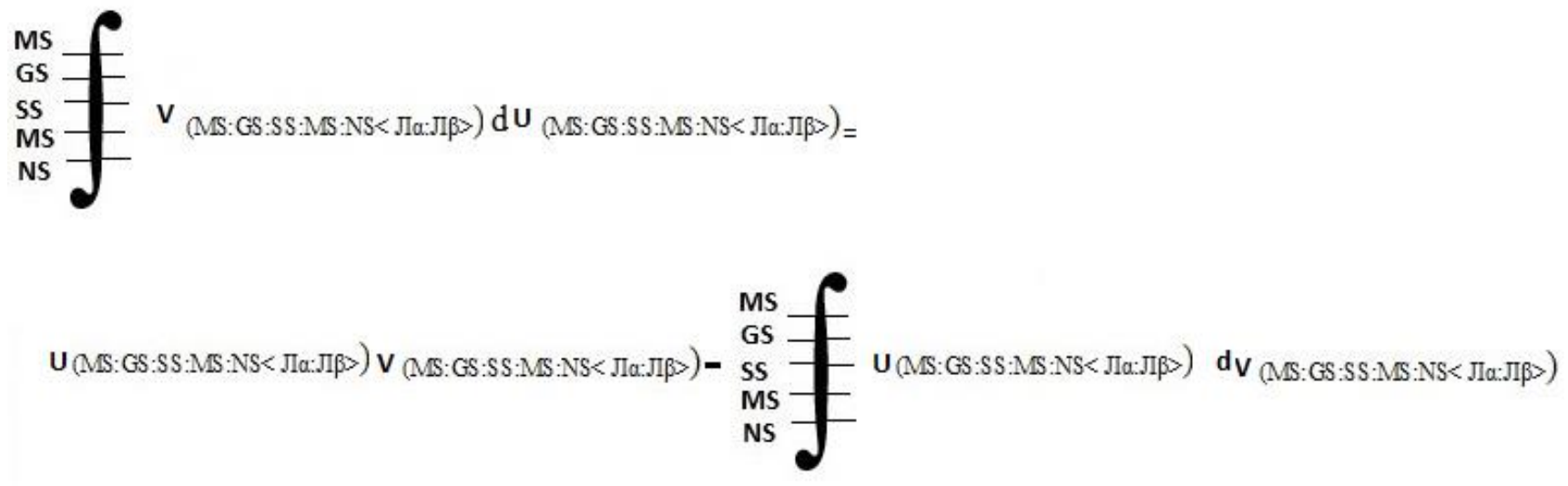

(9)

The dimension viability inter-connection rule (see Expression 10) is based on the connection of several integrations calculations in the same Dimension level (Space -S-) together in analysis without any restriction or parametric limitations. Hence, we have an alternative rule to increase different integrals calculations simultaneously in the same Dimension level at the same time. 


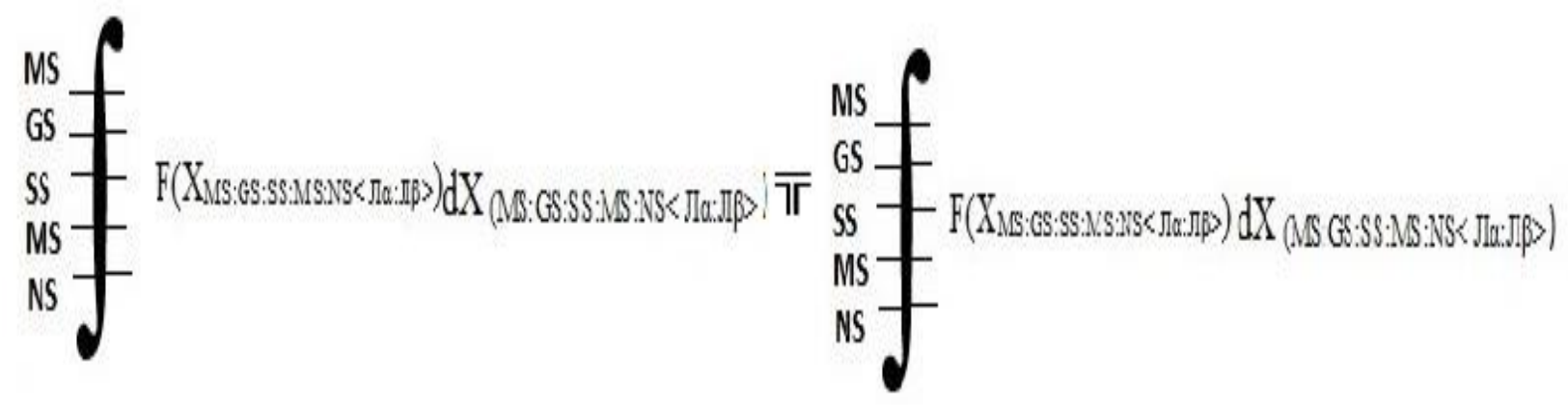

(10)

For the normalization interconnectivity rule (see Expression 11) is willing to connect a several number of integrations calculations from different Dimensions levels (Space levels $-\mathrm{S}-$ ) together in analysis without limitations of space and time. In this specific case, we have the possibility to join different integrals calculation on different dimensions at the same time and space respectively.

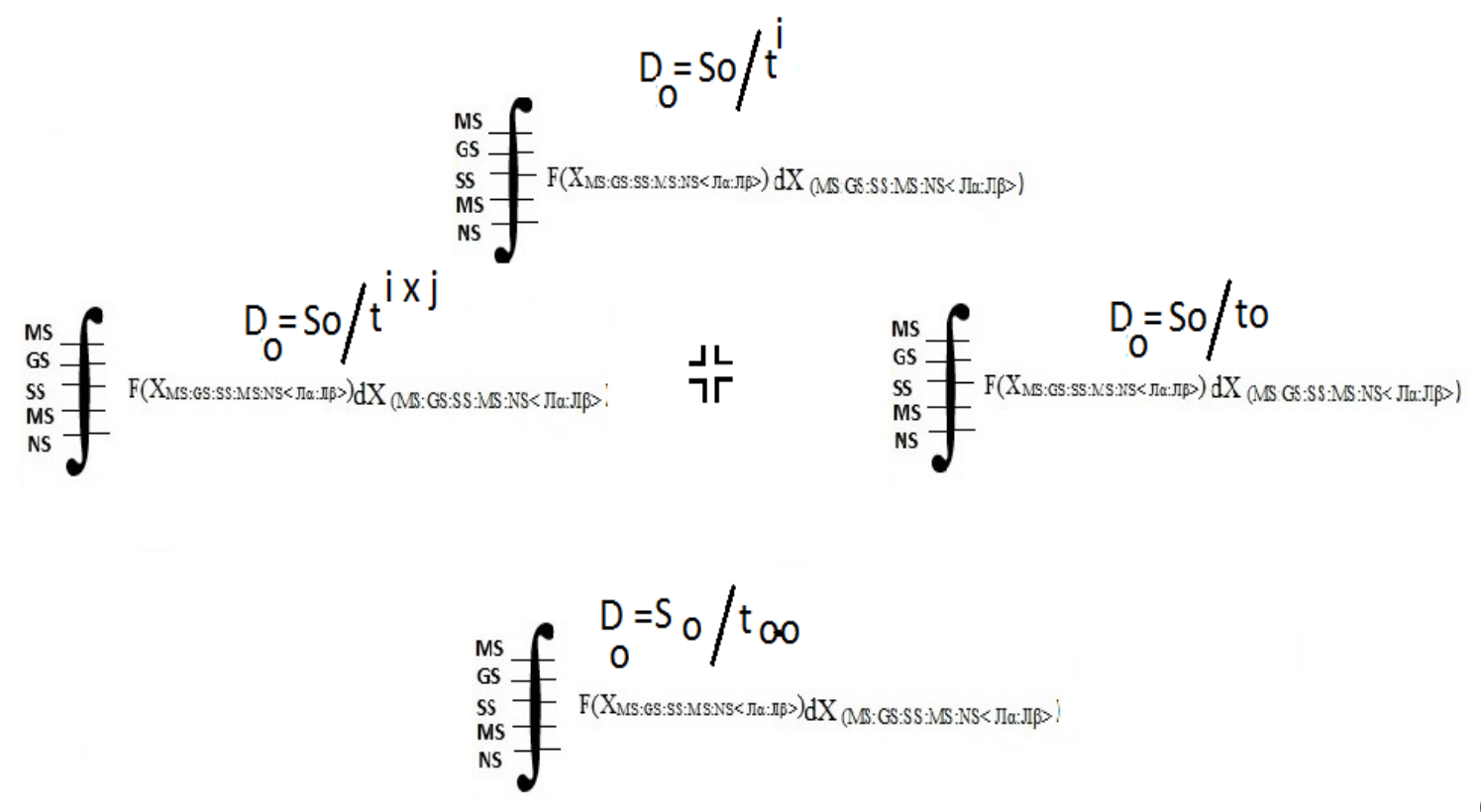


Finally, the definite multidimensional integrals (see Expression 12), the integral can be defined as any value exist between $\mathrm{JI}_{\alpha}$-Spaces levels $\wedge \mathrm{JI}_{\beta}$-Spaces levels. Where, the basic condition in the definite multidimensional integrals is that $\mathrm{JI}_{\alpha}=0 \wedge \mathrm{JI}_{\beta}=\infty$ but the $\mathrm{JI}_{\beta} \neq 0$. This condition is explicit in this specific case of definite integrals.

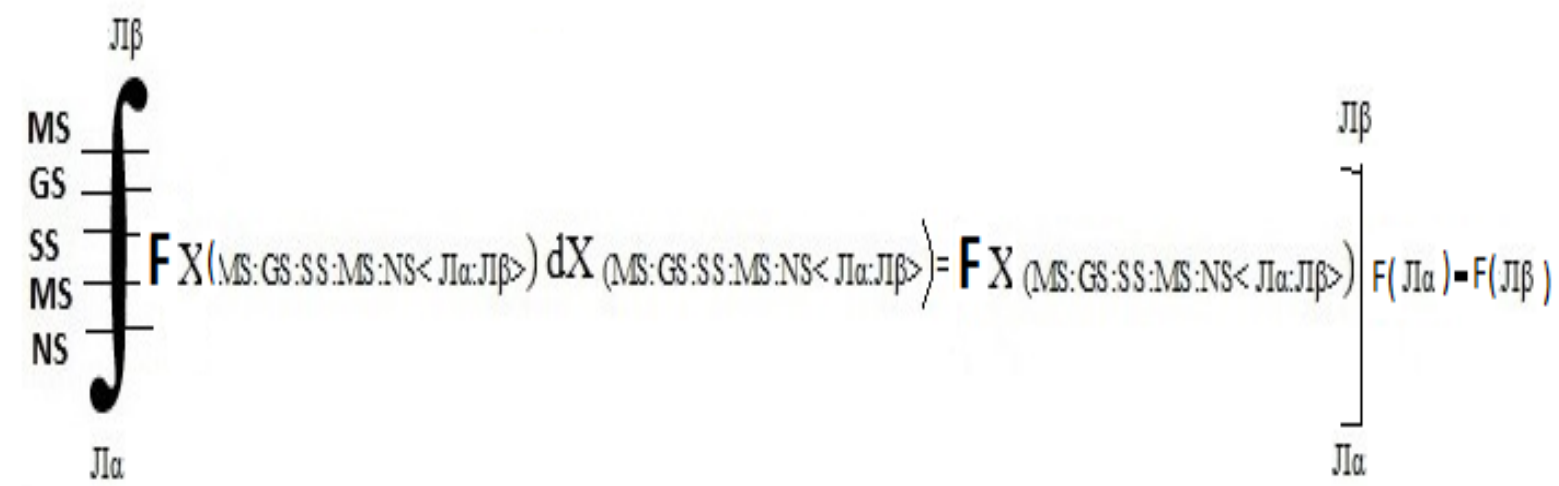

Moreover, the improper multidimensional integrals (see Expression 13), the integral can be defined as any value exist between $\left(-\infty, \mathrm{JI}_{\alpha}\right.$-Spaces levels $) \wedge\left(\mathrm{JI}_{\beta}-\right.$ Spaces levels, $\left.\infty+\right)$. Where, the basic condition in the improper multidimensional integrals is that $\mathrm{JI}_{\alpha}=-\infty \wedge \mathrm{JI}_{\beta}=\infty+$ but the $\mathrm{JI}_{\alpha} \vee \mathrm{JI}_{\beta}=(0, \infty+/-)$. This condition is explicit in this specific case of improper integrals.
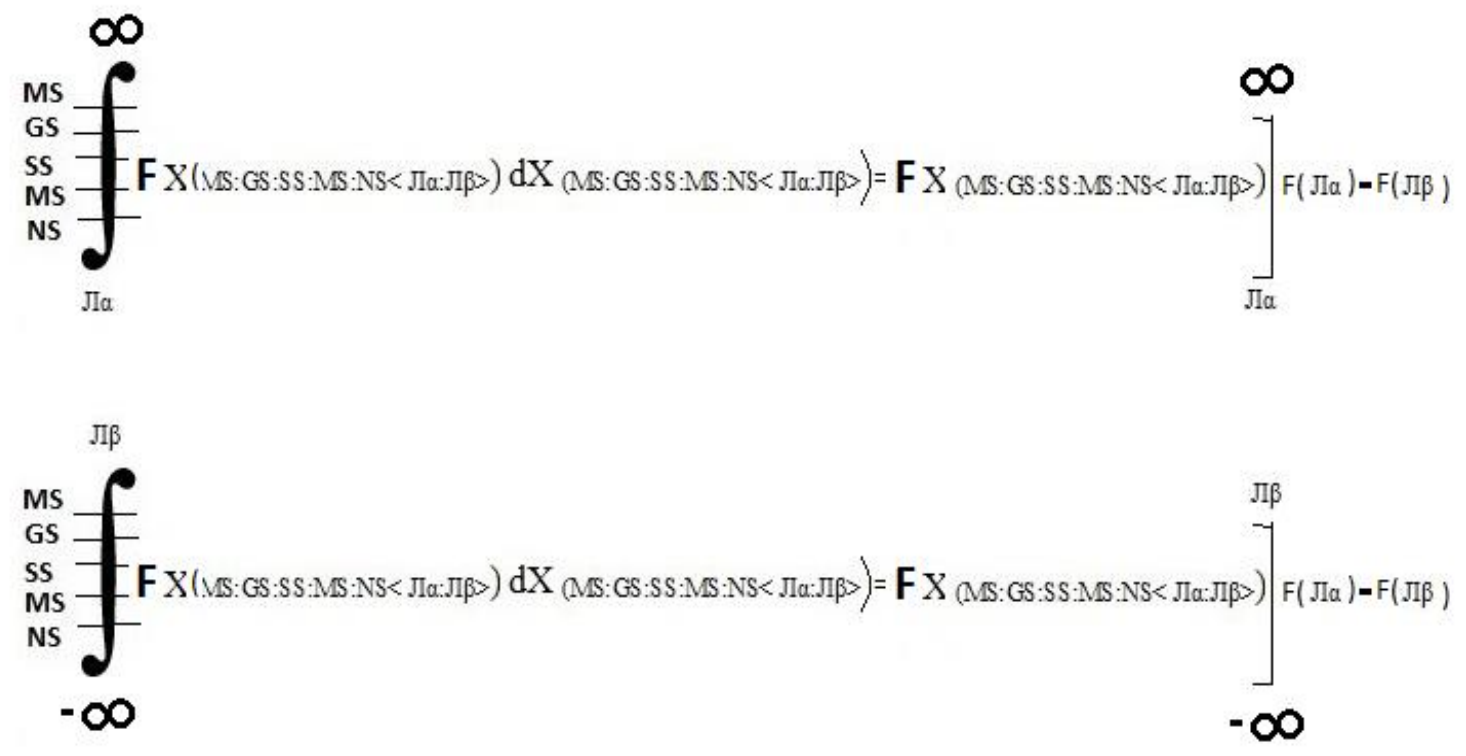
The Multidimensional Integral Calculus remark that the application of the Omnia Mobilis Assumption (Ruiz Estrada, 2011) plays an important role, because everything is moving in different speeds of time and spaces levels permanently. In figure 1, we can appreciate the Megasfinity Coordinate Spaces application. Finally, we can appreciate how integrals calculations in different Mega-Spaces levels (MS), General-Spaces levels (GS), Sub-Spaces levels (SS), Micro-Spaces levels (MS), and Nano-Space levels (NS) are able to calculate simultaneously.

Figure 1. Megasfinity Coordinate Spaces and Multidimensional Integrals
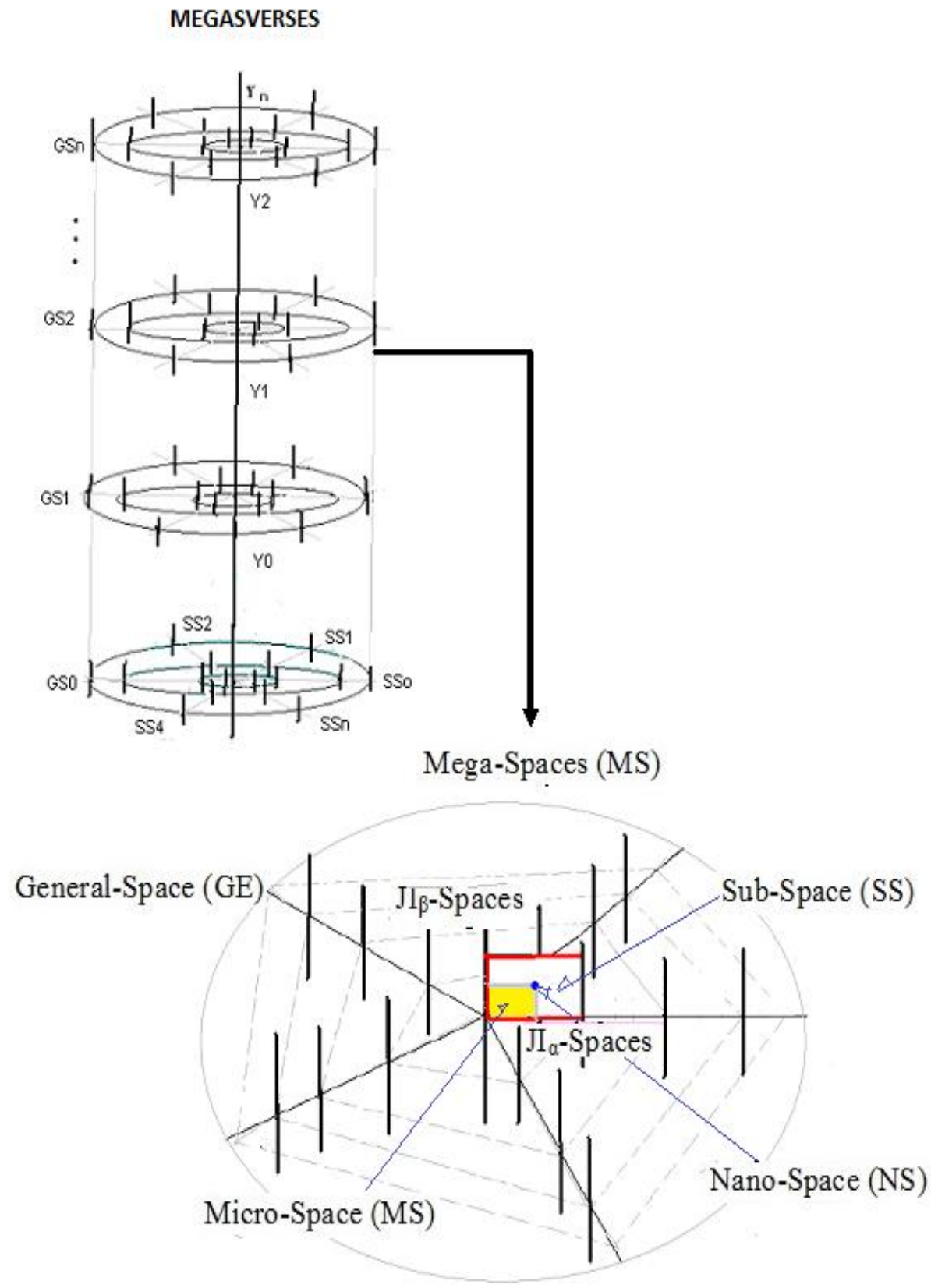


\section{Conclusion}

Basically, this paper concludes that it is possible to find a long number of integrals calculations in the Megasfinity Coordinate Spaces. The idea is to find ex-ante calculations or predifferentiation calculations at the same graphical space and time simultaneously. This multidimensional integration calculus is going to change the perception of time(s) and space(s) behaviour from a mathematical and graphical perspective. It is based on the use of a new set of rules for integrals (definite multidimensional integrals and improper multidimensional integrals) to generate a deep transformation in the study of integral calculus. According to our findings, the multidimensional integral calculus is going to help in the process to understand space and time behaviour (ex-ante) from a multi-dimensional point of view.

\section{References}

Abramowitz, M. and Stegun, I. A. (1972). (Eds.) Handbook of Mathematical Functions with Formulas, Graphs, and Mathematical Tables, 9th printing. New York: Dover, p. 258.

Hardy, G. H. and Wright, E. M. (1979). An Introduction to the Theory of Numbers, 5th ed. Oxford, England: Clarendon Press, pp. 13 \& 219.

Harris, J. W. and Stocker, H. (1998) "Cavalieri's Theorem." §4.1.1 in Handbook of Mathematics and Computational Science. New York: Springer-Verlag, p. 95, 1998.

Kern, W. F. and Bland, J. R. (1948) "Cavalieri's Theorem" and "Proof of Cavalieri's Theorem." $\$ 11$ and 49 in Solid Mensuration with Proofs, 2nd ed. New York: Wiley, pp. 25$27 \& 145-146$.

Ruiz Estrada, M.A. (2011). Policy Modeling: Definition, Classification, and Evaluation. Journal of Policy Modeling, 33(4), 523-536.

Ruiz Estrada, M.A. (2012) A new multidimensional approach for Mathematics and Physics. Malaysian Journal of Science, 31(2): 175-198. 